\title{
Treinamento em Primatologia no Brasil ${ }^{1}$
}

\author{
Milton Thiago de Mello²
}

O Brasil é o país do mundo que tem a maior diversidade de primatas. Até meados de 1994 existiam 16 gêneros, com 69 espécies, a maioria delas endêmicas e algumas ameaçadas de extinção (Fonseca et al., 1994). Os números exatos são ainda assunto para discussão entre especialistas por causa das subespécies e porque novas espécies estão sendo descobertas (quatro, desde 1990).

Apesar disso, como acontece na maioria dos países habitat, até 12 anos atrás o número de pessoas no Brasil com alguns conhecimentos sobre primatas era muito baixo, comparado com o de países sem primatas mas com primatologistas. Para preencher essa lacuna seria necessário ensinar ou treinar pessoas em Primatologia.

O treinamento em serviço era tradicional no Brasil (como em outros países habitat) para formação de profissionais especializados em Primatologia. Os pouquíssimos candidatos a treinamento eram agregados, sem nenhuma seleção, a uma das raras pessoas ativas, em geral usuários de primatas (geneticistas, psicólogos, microbiólogos, neurobiólocos) ou a conservacionistas existentes no país, a maioria deles autodidatas. Os resultados foram precários: menos de meia dúzia de pessoas com algum treinamento em campos limitados. Visitas esporádicas de primatologistas de outros países, para seus trabalhos de campo, não resultam na especialização dos nativos que, na maioria dos casos, eram simples ajudantes. É claro que umas poucas pessoas conseguiram fugir desté ćírculo vicioso e foram para o estrangeiro para fazer estágios ou cursos avançados de Mestrado ou Doutorado. Esșe era o quadro.

(i)

A fundação da Sociedade Brasileira de Primatologia (1979) não melhorou imediatamente a situação. Entretanto, durante o $1^{\circ}$ Congresso Brasileiro de Primatologia (Belo Horizonte, Minas Gerais, 1983), decidiu-se inverter a situação. Foi apresentado um trabalho (Mello, 1984a) apontando medidas a tomar para se conseguirem tão cedo quanto possível os especialistas necessários ao país. Uma lista adaptada daquele trabalho mostra a grande diversidade de assuntos que poderiam ser ensinados.
- Temas para estudos em Primatologia

Biologia Genética, hematologia, anatomia, fisiologia

Zoologia Taxonomia, evolução, morfologia, taxidermia

Ecologia

Etologia

Métodos de captura e transporte

Manejo de colônias Em cativeiro e semicativeiro

Quarentena, alimentação, higiene

Biologia da reprodução Em cativeiro e semicativeiro

Em condições naturais. Embriotecnologia

Produção zootécnica

Clínica veterinária

Profilaxia de zoonoses e outras doenças Patologia e terapêutica

Outras pesquisas

Plantas medicinais, produção de vacinas, monitoragem de pesticidas, biomodelos

Fez-se uma reunião de pessoas interessadas, entre elas Adelmar Coimbra Filho, Devra G. Kleiman, Bráulio Magalhães Castro, Milton Thiago de Mello e Russell Mittermeier. Chegou-se a uma concordância sobre quatro pontos básicos para futuras ações:

a) Necessidade de pessoal capacitado;

b) Treinamento por meio de cursos introdutórios formais regulares e anuais;

c) Cursos em diferentes partes do Brasil;

d) Professores que estivessem ativos no trabalho e de diferentes instituições do Brasil e do estrangeiro.

Essa necessidade de mais pessoas treinadas em diversos aspectos da Primatologia, principalmente conservação, motivou a Sociedade Brasileira de Primatologia, em colaboração com a Universidade de Brasília (UnB) e o "National Zoological Park/ Smithsonian Institution (NZP)", a organizar o Primeiro Curso de Especialização em Primatologia, de Pós-Graduação lato sensu, em 1983.

Tendo em vista que o número de primatologistas brasileiros, nessa ocasião, era muito pequeno, a cooperação de especialistas norte-americanos e britânicos foi muito importante. Recebeu-se valioso estímulo no NZP, a instituição que liderou o esforço internacional para salvar o mico-leão dourado (Leontopithecus rosalia) ameaçado de extinção. O permanente interesse e a participação de Devra Kleiman, do NZP, merecem destaque e agradecimentos.

A UnB se prontificou a oferecer Cursos de Especialização em Primatologia. A CAPES/Ministério da Educação foi a principal financiadora. Durante cada cur-

\footnotetext{
'Parte deste trabalho foi apresentada durante a reunião do "Grupo de Discussão sobre Programas de Pós-Graduação em Primatologia", presidido pela Profa Dorothy M. Fragaszy, Vice-Presidente para Sócios, International Primatological Society (IPS), XV Congresso da IPS, Bali, Indonésia, setembro de 1994.

IPS, Bali, Indonesia, setembro de 1994 . ricas da IPS e Professor da Faculdade de Veterinária, Universidade Federal Fluminense. Rua Vital Brazil Filho, 64 - CEP $24230-340$. Niterói, Rio de Janeiro, Brasijs
} 
so, muitas agências governamentais e organizações não governamentais (ONGs) deram forte apoio.

Desde a organização inicial decidido que cada curso teria curta duração (2-3 meses) mas a tempo integral. Com muita freqüência um dia de trabalho tinha mais de 18 horas. Cada curso possuía um tema diferente. Em alguns, mais ênfase na conservação e manejo em condições naturais em áreas selecionadas do Brasil. Todos tinham uma parte introdutória com aulas sobre conservação, biodiversidade, demografia, sistemática, legislação, etc.

Os Cursos de Especialização oferecidos pelo UnB foram os seguintes:

1 - 1983 - Conservação e Manejo

2 - 1984 - Taxonomia e Genética

3ํ - 1985 - Conservação e Manejo

4 - 1986 - Criação e Manejo de Primatas Neotropicais em Cativeiro

5 - 1987 - Conservação de Primatas em Áreas de Grande Impacto Ambiental

6ㅇ - 1989 - Patologia e Reprodução de Primatas Neotropicais

Eram dadas oportunidades para que os alunos participassem de reuniões e outras atividades científicas relacionadas com Primatologia e Conservação. Primatologistas e Conservacionistas do Brasil e de outros países foram convidados para fazer conferências ou palestras sobre suas respectivas especialidades.

Um aspecto importante para evitar endogenia ou "inbreeding" foi selecionar estudantes com passados profissionais, de instituições e de cidades diferentes. Os candidatos eram escolhidos depois de exame de seus currículos. É um prazer assinalar que mais de $70 \%$ dos alunos selecionados no seis cursos em jovens graduados com menos de dois anos de formados, que chegavam com imenso entusiasmo.

Em vez de dar ênfase a indivíduos, os cursos procuravam lidar com o grupo como unidade. Assim, existia uma grande troca de experiência e de conhecimentos profissionais. Cooperação, em vez de competição! Como os estudantes procediam de diferentes partes do Brasil e, algumas vezes, de outros países, também havia disseminação e intercâmbio de culturas. Os alunos de cada curso formaram, assim, fortes e duradouros laços de amizade, uma vez que tinham de trabaIhar em equipe e viver juntos no campo, às vezes em condições estressantes, em áreas remotas e difíceis do país.

Os professores e conferencistas eram selecionados entre pessoas ativas, com experiência e entusiasmo, de instituições brasileiras e estrangeiras, de acordo com o tema principal do curso. Procurava-se não repetir o mesmo professor mais de duas vezes, para evitar uma orientação uniforme para grupos sucessivos de estudantes.
Os locais para as aulas eram variados: nos primeiros cursos, eram poucos. Depois novos foram escoIhidos, em parte devido aos esforços de ex-alunos que tinham ido trabalhar nesses lugares: o almejado efeito multiplicador.

A relação a seguir mostra os Cursos de Especialização em Primatologia e os principais locais dos Estados onde foram dadas as aulas:

DISTRITO FEDERAL - Fazenda Água Limpa/UnB, Jardim Zoológico de Brasília, Reserva Biológica de Águas Emendadas, Parque Nacional de Brasília, Jardim Botânico de Brasília, Fazenda Sucupira, Reserva Ecológica do IBGE: Primatas do Cerrado (Callithrix penicillata, Cebus apella, Alouatta caraya). Centro Nacional de Recursos Genéticos (Brasília): Genética de animais selvagens.

RIO DE JANEIRO - Centro de Primatologia do Rio de Janeiro (Guapimirim): Criação de espécies de primatas da Mata Atlântica ameaçadas de extinção (gen. Leontopithecus, Callithrix, Cebus, Brachyteles); projeto mico-leão dourado. Reserva Biológica de Poço das Antas (Silva Jardim): Projeto mico-leão dourado. Museu Nacional do Rio de Janeiro: Primatas preservados. Jardim Zoológico do Rio de Janeiro: Primatas vivos; criação em cativeiro. Faculdade de Veterinária/ UFF (Niterói): Medicina Veterinária de animais selvagens, inclusive primatas; $2^{\circ}$ Simpósio de Medicina Veterinária de Animais Silvestres. Fundação Oswaldo Cruz (FIOCRUZ), Centro de Primatas; Criação de rhesus (Macaca Mulatta) e macaco de cheiro (Saimiri sciureus).

MINAS GERAIS - Estação Biológica de Caratinga/ FBCN, Fazenda Montes Claros (Caratinga): Conservação de primatas de Mata Atlântica (Brachyteles arachnoides, Callithrix flaviceps, Alouatta fusca e Cebus apella). Parque Estadual do Rio Doce (Campolina): Primatas da Mata Atlântica ( $B$. aracnoides, C. apella).

SÃO PAULO - Reserva Estadual do Morro do Điabo (Teodoro Sampaio): Resgate de L. chrysopygus. Reunião Científica Regional do ICLAS sobre animais de laboratório (Águas de Lindóia): Confêrências sobre primatas e conservação na América Latina, Fundação da Sociedade Latino Americano de Primatologia, $2^{\circ}$ Congresso Brasileiro de Primatologia (Campinas): Conferências sobre primatas e conservação. Parque Zoológico de São Paulo, Instituto Butantã, Instituto de Botânica, Exotiquarium (São Paulo): Conferência e demonstrações sobre primatas e conservação de animais selvagens.

PARÁ - Museu Paraense Emílio Goeldi (Belém): Primatas da Amazônia vivos e preservados. Centro $\mathrm{Na}$ cional de Primatas (Belém): Criação de primatas da Amazônia. Departamento de Genética e de Psicologia/UFPA (Belém): Pesquisas sobre genética e com- 
portamento de primatas da Amazônia. Instituto Evandro Chagas (Belém): Pesquisas médicas com primatas da Amazônia.

AMAZONAS - Usina Hidroelétrica de Balbina/ ELETRONORTE (Balbina): Conservação de primatas em área de grande impacto ambiental; resgate de primatas. Estação Ecológica Lago do Mamirauá (Tefé): Conservação de primatas da Amazônia (Cacajao calvus, Saimiri vanzolini, Alouatta seniculus). Centro de Instrução de Guerra na Selva/Ministério do Exército (Manaus): Sobrevivência na Floresta Amazônia. Instituto Nacional de pesquisas da Amazônia (Manaus): Primatas e conservação em geral na Amazônia. Instituto de Medicina Tropical da Amazônia (Manaus): Pesquisas com primatas.

Embora os cursos começassem na UnB, os estudantes sempre viajam para outras partes do país, onde encontravam novos primatas (humanos e não humanos) em seus habitats e também aumentavam seu conhecimento sobre o "Brasil real", muito diferente do "Brasil oficial" encontrado na maioria das universidades, instituições de pesquisa e agências governamentais das grandes cidades. Além disso, observavam in loco onde o trabalho estava sendo feito.
Depois da conclusão dos cursos, a UnB fornecia para cada estudante um certificado, no verso do qual constava uma lista dos professores e dos assuntos das aulas. Os alunos do $1^{\circ}$ Curso (1983) também receberam um certificado do NZP. Para obter o certificado cada estudante deveria apresentar até dois meses após a conclusão do curso, um relatório final das observações feitas e sugestões para a melhoria dos próximos cursos. A leitura desses relatórios, feitos individualmente e sem regras firmes para sua elaboração, permitia a verificação dos interesses e das tendências de cada estudante. Os relatórios dos seis cursos constituem uma coleção impressionante de 12 grandes volumes que estão no acervo de publicações sobre Primatologia da Biblioteca da UnB, como boa fonte de informações. Alguns eram tão detalhados e precisos que partes deles foram usadas pelos seus autores como base para trabalhos apresentados em congressos ou publicados em revistas científicas.

Em alguns cursos os estudantes tiveram a oportunidade de participar de acontecimentos relacionados com Primatologia e reuniões científicas. Por exemplo: a) Chegada no Centro de Primatologia do Rio de Janeiro, de 15 exemplares mico-leão dourado

As Tabelas 1 e 2 resumem as especialidades de graduação e os locais de origem dos estudantes. Esta última, importante num país de dimensões continentais como o Brasil.

Tabela 1 - Cursos de Especialização em Primatologia. Formação acadêmica dos alunos

\begin{tabular}{|c|c|c|c|c|c|c|c|}
\hline Graduação & $\begin{array}{c}\text { 1ํ Curso } \\
1983 \\
\text { Conservação }\end{array}$ & $\begin{array}{c}\text { 2o Curso } \\
1984 \\
\text { Taxonomia e } \\
\text { Genética }\end{array}$ & $\begin{array}{c}\text { 3o Curso } \\
1985 \\
\text { Criação em }\end{array}$ & $\begin{array}{c}\text { 4ํ Curso } \\
1986 \\
\text { Conservação } \\
\text { Cativeiro }\end{array}$ & $\begin{array}{c}5^{\circ} \text { Curso } \\
1987\end{array}$ & $\begin{array}{c}\text { 60 Curso } \\
1989 \\
\text { Patologia e } \\
\text { Reprodução }\end{array}$ & Total \\
\hline Biologia & 7 & 3 & 5 & 6 & $8^{a}$ & 1 & 30 \\
\hline $\begin{array}{l}\text { Medicina } \\
\text { Veterinária }\end{array}$ & 2 & 3 & $2^{\mathrm{a}}$ & $8^{b}$ & 4 & $12^{d}$ & 31 \\
\hline Psicologia & 1 & 1 & 1 & 2 & & & 5 \\
\hline Ecologia & 1 & & 1 & & $2 c$ & & 4 \\
\hline Arqueologia & & 1 & & & & & 1 \\
\hline $\begin{array}{l}\text { Antropologia } \\
\text { Físico }\end{array}$ & & & 1 & & & 1 & \\
\hline Zootecnia & & & 1 & & & & 1 \\
\hline \multirow{2}{*}{$\begin{array}{l}\text { Engenharia } \\
\text { Florestal }\end{array}$} & & & & & 1 & & 1 \\
\hline & 11 & 8 & 11 & 16 & 15 & 13 & 74 \\
\hline
\end{tabular}
a - 1 da Colônia
b - 1 do Peru
c - 1 do México
1 do México
d - 1 do México
1 do Uruguai
1 da Argentina


Tabela 2 - Cursos de Especialização em Primatologia. Procedência dos alunos

\begin{tabular}{|c|c|c|c|c|c|c|c|}
\hline Origem & $\begin{array}{c}1^{\circ} \\
1983\end{array}$ & $\begin{array}{c}2^{\circ} \text { Curso } \\
1984\end{array}$ & $\begin{array}{c}3^{\circ} \text { Curso } \\
1985\end{array}$ & $\begin{array}{c}4^{\circ} \text { Curso } \\
1986\end{array}$ & $\begin{array}{c}5^{\circ} \text { Curso } \\
1987\end{array}$ & $\begin{array}{c}6^{\circ} \text { Curso } \\
1989\end{array}$ & Total \\
\hline $\begin{array}{l}\text { BRASIL (Estados) } \\
\text { Amazonas } \\
\text { Pará } \\
\text { Rio Grande do Norte } \\
\text { Paraíba } 1 \\
\text { Pernambuco } \\
\text { Alagoas } \\
\text { Bahia } \\
\text { Espírito Santo } \\
\text { Rio de Janeiro } \\
\text { Minas Gerais } \\
\text { São Paulo } \\
\text { Mato Grosso do Sul } \\
\text { Rio Grande do Sul } \\
\text { Distrito Federal } \\
\text { OUTROS PAíSES } \\
\text { Argentina } \\
\text { Colômbia } \\
\text { México } \\
\text { Peru } \\
\text { Uruguai }\end{array}$ & $\begin{array}{l}2 \\
1 \\
1 \\
3\end{array}$ & $\begin{array}{l}2 \\
1 \\
1 \\
2\end{array}$ & $\begin{array}{l}1 \\
1 \\
3 \\
1 \\
1\end{array}$ & $\begin{array}{l}1 \\
2 \\
1 \\
1\end{array}$ & $\begin{array}{l}1 \\
1 \\
1 \\
3 \\
1 \\
2 \\
1 \\
1 \\
2\end{array}$ & $\begin{array}{l}1 \\
1 \\
1 \\
1 \\
3 \\
1 \\
1 \\
2 \\
1 \\
1 \\
1 \\
1\end{array}$ & $\begin{array}{c}1 \\
7 \\
3 \\
\\
3 \\
\\
4 \\
1 \\
13 \\
7 \\
9 \\
2 \\
5 \\
9\end{array}$ \\
\hline Total & 11 & 8 & 11 & 16 & 15 & 13 & 74 \\
\hline
\end{tabular}

(Leontopithecus rosalia) procedentes do NZP constituindo a primeira fase do "Projeto Mico-Leão Dourado" (1ㅇ Curso, 1983);

b) $2^{\circ}$ Congresso Brasileiro de Primatologia, Campinas, São Paulo ( $3^{\circ}$ Curso, 1985);

c) Fundação da Sociedade Latino Americana de Primatologia, durante a Reunião Científica Regional do ICLAS (Internacional Council of Laboratory Animal Science), Águas de Lindóia, São Paulo (4ำ Curso, 1986);

d) 2o Simpósio de Medicina Veterinária de Animais Silvestres, Faculdade de Veterinária/UFF, Niterói, Rio de Janeiro (4\% Curso, 1986);

e) Inauguração da Estação Ecológica Lago do Mamirauá, perto de Tefé, Amazonas, sob a direção dọ Dr. José Márcio Ayres (5 Curso, 1987).

Num outro nível de atividades, para difundir conhecimentos e resultados de pesquisas sobre Primatologia no Brasil, a Sociedade Brasileira de Primatologia organizou os Congressos Brasileiros de Primatologia. Até agora foram realizados sete em diferentes partes do país $(1983,1985,1987,1989,1990,1994)$. Os Anais dos cinco primeiros foram publicados (Mello, 1984b; Mello, 1986; Rylands e Bernardes, 1991; Yamamoto e Souza, 1993). Durante o 6o Congresso, realizado em julho de 1994, no Rio de Janeiro, 64 trabalhos foram apresentados por 91 autores, em 2 conferências, 3 simpósios e 4 sessões de comunicações orais. $07^{\circ}$ Congresso foi realizado em Natal, Rio Grande do Norte, em julho de 1995.

Em 1988, o Brasil sediou o XII Congresso da "International Primatological Society", em Brasília. Foi o primeiro congresso da IPS num país Neotropical habitat de primatas. Diversos trabalhos foram apresentados por primatologistas brasileiros, alguns destes exalunos dos cursos da UnB.

Sobre as atividades de seguimento dos ex-alunos, como eles foram altamente motivados por professores entusiastas e puderam ter uma visão abrangente da Primatologia, a maioria se inscreveu em cursos avançados para obter o diploma de Mestre ou Doutor, no Brasil ou no estrangeiro. Todos eles estão agora trabalhando com primatas, conservação ou asșuntos correlatos. Um resultado impressionante, a partir de tão pouco.

Assim, num curto espaço de tempo (12 anos), fortes grupos de primatologistas foram constituídos e não pessoas isoladas. O que é mais importante: os 74 profissionais egressos dos cursos da UnB também agiram como catalisadores para outras pessoas transmitindo interesse e entusiasmo: outra vez o efeito multiplicador. Como resultado de todas essas circunstâncias, a Sociedade Brasileira de Primatologia tem hoje mais de 300 sócios. 
O Brasil é agora praticamente o único país habitat de primatas Neotropicais que efetuou um programa regular de treinamento de pessoal, compatível com seu grande patrimônio primatológico. É um exemplo que certamente pode ser seguido em outros países.

Fora do Brasil, têm havido oportunidades para treinamento em serviço ou como parte de cursos de pósgraduação em outros assuntos, oferecidos por diversos instituições, principalmente na Europa e nos EEUU. A Primatologia, entretanto, não é o assunto principal específico. A maioria está relacionada a conservação, criação em cativeiro e comportamento de animais selvägens em geral. Referências podem ser encontradas em catálogos de universidades. Uma boa lista foi publicada em 1978: "Directory of Graduate Programs in Primatology and Primate Research" (Anônimo, 1978). Num trabalho apresentado durante o III Congresso Brasileiro do Primatologia (Juiz de Fora, Minas Gerais, 1987 (Waugh, 1991), foi mencionada a necessidade de treinamento de pessoal para a criação em cativeiro e a conservação de primatas, detalhando os programas oferecidos pelo "Jersey Wildlife Preservation Trust" (JWPT) e citando alguns outros. Entretanto, todos eles eram relacionados com animais silvestres em geral, não especificamente primatas. É .curioso notar que num Congresso de Primatologia realizado no Brasil e depois de quatro curso de especialização bem sucedidos efetuados na UnB; alguns deles com a participação do Dr. Jeremy C. Mallinson, eminente primatologista do JWPT, nenhuma referência fosse feita a esses cursos.

Além dos congressos e cursos, um fator que também contribuiu para o treinamento em Primatologia no Brasil foi o interesse pelo mico-leão dourado (Leontopityhecus-rosalia) e pelo muriqui (Brachyteles arachnoides), nos Estados do Rio de Janeiro e Minas Gerais, respectivamente. Por causa disso, algumas pessoas têm recebido treinamento em serviço enquanto fazem suas pesquisas, quase sempre como parte de um programa de pós-graduação, inicialmente sob orientação de Devra Kleiman e Karen Strier, respectivamente. O papel de organizações não governamentais (ONGs) brasileiras e estrangeiras tem sido importante para isso, dando apoio para pesquisas de campo.

Às vezes algumas pessoas perguntam se não foram encontradas dificuldades para organizar os cursos, convidar e reunir professores, selecionar estudantes, conseguir bolsas, auxílios e apoio, contornar a burocracia, providenciar transporte, alojamento e comida para estudantes e professores no campo, etc. Naturalmente que sim! Mas nada se compara com o prazer de contribuir para a formação de uma geração de jovens primatologistas e para o desenvolvimento da Primatologia no Brasil.

\section{Agradecimentos}

Atividades como as descritas, não poderiam ser efetuadas sem ajuda de muitas pessoas e instituições. É com grande prazer que são feitos agradecimentos aos professores, pesquisadores e pessoal técnico-administrativo das dezenas de instituições que participaram dos seis cursos oferecidos pela UnB. No total, foram centenas de pessoas das instituições relacionadas adiante e já mencionadas neste trabalho. Por isso, não são todas nominadas. Entretanto, merecem menção as seguintes Adelmar F. Coimbra Filho, Alcides Pissinatti, Roberto Rocha e Silva, Claudio B. Valladares Pádua (Centro de Primatologia do Rio de Janeiro), Célio Murilo de Carvalho Valle, Anthony Brome Rylands, I. mar Bastos Santos, Gustavo B. Fonseca (Departamento de Zoologia/ICB, Universidade Federal de Minas Gerais).

Desejo destacar a Dra. Maria lolita Bampi, aluna do 2 ․ Curso, ex-colega na UnB, ex-responsável pelo Centro de Primatologia da UnB e agora Diretora de Fauna do IBAMA (Instituto Brasileiro do Meio Ambiente e dos Recursos Naturais Renováveis). Durante mais de 10 anos tenho tido a satisfação de contar com sua colaboração, entusiasmo e amizade. Instituições - Universidade de Brasília (UnB); Universidade Federal Fluminense (UFF); Universidade Federal de Minas Gerais (UFMG); Universidade Federal do Rio de Janeiro (UFRJ); Universidade Federal do Pará (UFPA); Universidade de São Paulo (USP); Universidade Estadual de Campinas (UNICAMP); Escola Paulista de Medicina; Fundação Coordenação de Aperfeiçoamento de Pessoal de Nível Superior (CAPES/Ministério da Educação); Conselho Nacional do Desenvolvimento Científico e Tecnológico (CNPq); Financiadora de Estudos e Projetos (FINEP); Sociedade Brasileira de Primatologia (SBPr); Fundação Brasileira para a Conservação da Natureza (FBCN); Centro de Primatologia do Rio de Janeiro (CPRJ/FEEMA); Instituto Brasileiro de Desenvolvimento Florestal (IBDF), agora Instituto Brasileiro do Meio Ambiente e dos Recursos Naturais Renováveis (IBAMA); Fundação Zoobotânica do Distrito Federal (FZ/DF): Jardim Zoológico, Jardim Botânico, Reserva Biológica de Águas Emendadas; Museu Paraense Emílio Goeldi/Belém; Centro Nacional de Primatas/Belém; Instituto Evandro Chagas/Belém; Centro Nacional de Recursos Genéticos (CENARGEN/ EMBRAPA/Brasília); Reserva Ecológica do Instituto Brasileiro de Geografia e Estatística (IBGE/Brasília); Museu Nacional (MN/UFRJ); Fundação Parque Zoológico do Rio de Janeiro (RioZoo); Departamento de Florestas de Minas Gerais; Instituto de Florestas de São Paulo; Instituto Nacional de Pesquisa da Amazônia (INPA/CNPq); Instituto de Medicina Tropical da Amazônia (IMTA/Manaus); Centro de Instrução de Guerra na Selva (CIGS/Ministério do Exército/Manaus); Centrais Elétricas do Norte (ELETRONORTE); Sociedade 
Brasileira de Medicina Veterinária (SBMV); Conselho Federal de Medicina Veterinária (CFMV); Companhia Energética de São Paulo (CESP); Secretaria do Meio Ambiente (SEMA); Fundação Pró-Natureza (FUNATURA); Parque Nacional de Brasília/IBAMA; Reserva Biológica de Poço das Antas/IBAMA; Fundação Instituto Oswaldo Cruz (FIOCRUZ); Instituto Butantã; Faculdade de Medicina Veterinária e Zootecnica/USP; Simba Safari/São Paulo; Exotiquarium/São Paulo; Instituto de Botânica/USP.

Professores e Conferencistas Estrangeiros - Devra G. Kleiman, James M. Dietz, Lou Ann Dietz, Margareth O'Connell e Mitchell Bush (National Zoological Park/ Smithsonian Institution, Washington, DC, USA); John Hearn, David Abbott (The Zoological Society of London e Wisconsin Regional Primate Research Center, USA); Charles T. Snowdon e Karen Strier (University of Wisconsin, USA); Robert Nelson (Society of Friends of the National Zoological Park, USA); Murray F. Fowler (University of California-Davis, USA); Scott M. Lindberg (Verlhiac Primate Center, França e Parque Nacional. de Brasília/IBAMA); William R. Kingston (Organização Panamericana da Saúde/Centro Nacional de Primatas, Belém); Jeremy J. C. Mallinson (Jersey Wildlife Preservation Trust, Grã-Bretanha); Boris A. Lapin (Institute of Experimental Pathology and Therapy, Sukhumi, URSS); Jaime Alfonso Umaña A. (Instituto Nacional de Salud e Universidad Nacional de Colombia, Bogotá); Russell A. Mittermeier (Conservation International).

\section{Referências Bibliográficas}

ANÔNIMO - Directory of Graduate Programs in Primatology and Primate Research. Lab. Primate Newsletter, v. 17, n. 4, 1978.

FONSECA, G.A.B., RYLANDS, A.B., COSTA, C.M.R., MACHADO, R.B, e LEITE, Y.L.R. (eds.) (1994). Li- vro Vermelho dos Mamíferos Brasileiros Ameaçados de Extinção, Fundação Biodiversitas, Belo Horizonte: $459 \mathrm{pp}$.

MELLO, M.T. de (1984a) - Treinamento de Pessoal em Primatologia. In Mello, M.T. de (ed.,), A Primatologia no Brasil, Anais $1^{\circ}$ Congresso Brasileiro Primatologia, Belo Horizonte, 1983. Soc. Brasileira Primatologia, pp. 373-386.

MELLO M.T. de (ed.) (1984b) - A Primatologia no Brasil, Anais $1^{\circ}$ Congresso Brasileiro Primatologia, Belo Horizonte, 1983. Soc. Brasileira Primatologia, 402 pp.

MELLO, M.T. de (ed.) (1986) - A Primatologia no Brasil-2, Anais $2^{\circ}$ Congresso Brasileiro. Primatologia, Campinas, 1985. Soc. Brasileira Primatologia, 530 pp.

RYLANDS, A.B. e BERNARDES, A.T. (eds.) (1991). A Primatologia no Brasil-3, Anais III. Congresso Brasileiro Primatologia, Juiz de Fora, 1987 e Anais IV Congresso Brasileiro Primatologia, João Pessoa, 1989. Soc. Brasileira Primatologia, 459 pp.

WAUGH, D.R. (1991) - Training opportunities for the captive breeding and conservation of primates. In Rylands, A.B. e Bernardes, A.T. (eds.) A Primatologia no Brasil-3, Anais III Congresso Brasileiro Primatologia, Juiz de Fora, 1987 e Anais IV Congresso Brasileiro Primatologia, João Pessoa, 1989. Soc. Brasileira Primatologia, pp. 393-404.

YAMAMOTO, M.E. e SOUZA, M.B.G. de (eds.) (1993). A Primatologia no Brasil-4, Anais V. Congresso Brasileiro Primatologia, Salvador, 1990. Soc. Bras. Primatologia, $327 \mathrm{pp}$.

\section{Revista Brasileira de Ciência Veterinária Publicação Quadrimestral da Faculdade de Veterinária da UFF}

\section{Para Anunciar}

